\title{
Study on Transition of Primary Energy Structure and Carbon Emission Reduction Targets in China Based on Markov Chain Model and GM $(1,1)$
}

\author{
Feng $\operatorname{Ren}^{1}$ and Lihong $\mathbf{G u}^{2}$ \\ ${ }^{1}$ School of Business and Administration, North China Electric Power University, Baoding, Hebei Province 071003, China \\ ${ }^{2}$ Foreign Language Teaching Department, Hebei Finance University, Baoding, Hebei Province 071051, China \\ Correspondence should be addressed to Feng Ren; renfeng2002@126.com
}

Received 14 June 2016; Accepted 7 November 2016

Academic Editor: Marcello Pellicciari

Copyright (c) 2016 F. Ren and L. Gu. This is an open access article distributed under the Creative Commons Attribution License, which permits unrestricted use, distribution, and reproduction in any medium, provided the original work is properly cited.

\begin{abstract}
The improvement of the primary energy structure has been considered as one of the important measures to achieve the carbon emissions reduction targets in China. This current paper constructed a Markov chain model, which was used to forecast the transition of primary energy structure. GM $(1,1)$ model and a linear regression model were used to predict the total energy consumption in 2020 and 2030. Then, the $\mathrm{CO}_{2}$ emissions intensity was calculated, and the realization of carbon emissions reduction targets in China was analyzed. The findings indicated that (1) China's nonfossil energy share in primary energy cannot be achieved naturally. (2) Part of the carbon emissions intensity in China's commitments was not binding actually. (3) The realization of the carbon emissions peak and the reduction target of carbon emissions intensity in 2030 would need the policy intervention. In the last part of this paper, policy recommendations on carbon emissions reduction in China were provided.
\end{abstract}

\section{Introduction}

China's primary energy consumption and $\mathrm{CO}_{2}$ emissions have been widely concerned by the international community. With the rapid development of economy and society, China's primary energy demands grow rapidly. Coal, with high carbon property, dominates in China's energy consumption with a share of $70 \%$. In the decade after 2000, China's coal consumption and $\mathrm{CO}_{2}$ emissions were increasing at an average annual rate of $9 \%$. China has become the largest coal consumer and $\mathrm{CO}_{2}$ emitter in the world [1].

But, China has not always been the world's biggest emitter. From the beginning of the industrial era, the US emitted four times more $\mathrm{CO}_{2}$ than China on a cumulative basis. For this reason, during climate negotiations, China has always been a fierce advocate of the "common but differentiated responsibilities," which calls for developed countries to take on more ambitious and quantified commitments than developing countries [2]. Today though, this issue is controversial because China's unprecedented economic expansion pushes it out of the "developing country" list. If we consider emissions from 1990 to today, China has almost caught up with the US total.

The rapid increase in primary energy consumption and $\mathrm{CO}_{2}$ emissions has led to the deterioration in the ecological environment and the people's livelihood in China. The direct reasons for the Chinese authorities to urgently improve the primary energy structure are most likely as follows.

(a) The consideration of energy conservation and energy efficiency: China's energy supply has become one of the strategic issues related to energy security and national security. China's energy consumption was growing at an alarming rate. In 1999, China's total energy consumption was about half of United States' consumption. In 2009, China consumed 2.252 billion Ton Oil Equivalents (TOES), while the United States consumed 2.170 billion TOES. It can be predicted that, with the gradual completion of China's industrialization and urbanization process, the energy demand 
will increase dramatically. So, the Chinese authorities have to consider energy conservation and energy efficiency in order to achieve sustainable development of economy and society.

(b) The consideration of carbon reduction: the Chinese authorities have promised to reduce carbon emissions intensity and formulated the special national plan, "the national response to climate change rule (2014-2020)." At the Paris Climate Conference, the Chinese government promised to peak its $\mathrm{CO}_{2}$ emissions and let $\mathrm{CO}_{2}$ emissions intensity fall by $60-65 \%$ by 2030 from 2005 level [3]. However, special national conditions determine that China's energy supply will rely on coal for a long period of time in the future. So, the situation of carbon emissions for China will still be very grim. Obviously, the improvement of primary energy structure will help to realize the target of China's carbon emissions reduction.

(c) The consideration of ecological protection: for a long time in the past, the Chinese governments at all levels devoted themselves to economic development and did not pay enough attention to ecological protection. Nowadays, China's ecological environment has been deteriorated very seriously. For example, in the winter of 2015, the whole northern China and even most parts of the country experienced severe haze weather. Beijing, the capital of China, issued its highest smog alert of the year. The Air Quality Index (AQI) in Beijing stood at levels "beyond index" just as the governments of more than 190 countries were meeting at the 2015 Paris Climate Conference in an attempt to forge a new global agreement on climate change. Allegedly, the haze was ascribed to emissions from coal combustion. As binding targets, environmental governance objectives have been listed in the 13th Five-Year Plan of China. So, the Chinese government is eager to reduce the proportion of coal to realize the ecological protection target.

(d) The consideration of people's livelihood improvement: application of small coal stove seriously pollutes the indoor living environment in China. Coal combustion emits carbon dioxide, sulfur dioxide, carbon monoxide, and other toxic and harmful gases. People living in this environment extremely likely suffer from respiratory inflammation, chronic bronchitis, bronchial asthma and emphysema, and other diseases. In addition, sulfur dioxide is also an important cause of cardiovascular diseases, such as coronary heart disease, arteriosclerosis, and hypertension. From the perspective of fairness and development results sharing, the Chinese authorities should provide alternative energy sources of coal and improve the living environment for these inhabitants.

In short, considering energy conservation, energy efficiency, carbon emissions reduction, ecological protection, and people's livelihood improvement, the Chinese authorities should take great efforts to improve the primary energy structure.

Distinctly, the Chinese authorities have noticed the seriousness of the problem, urgently prepared to improve the primary energy structure, and made solemn commitments to the whole world. The commitments came in the much awaited Intended Nationally Determined Contribution
(INDC) that China presented ahead of the UN climate talks in Paris (COP21). The Chinese authorities' commitments can be summed up to three targets: target 1, aiming to increase the share of nonfossil fuel sources in primary energy consumption to about $15 \%$ and $20 \%$ by 2020 and 2030, respectively; target 2, peaking its $\mathrm{CO}_{2}$ emissions around 2030 (about 12 billion tons); target 3, reducing $\mathrm{CO}_{2}$ emissions intensity by $40-45 \%$ and $60-65 \%$ from 2005 level by 2020 and 2030, respectively. Some studies have analyzed these issues from the perspectives of the whole or of different industries and the results have differed greatly [4-17]. For example, Stern and Jotzo and Dai et al. believed that the carbon reduction target was a challenging task $[15,16]$, but Cansino et al. drew a conclusion that China's emissions reduction task can be achieved without extra effort [17]. However, can these targets really be achieved naturally? How to achieve these targets? These are exactly what we are interested in.

Studies on energy intensity and carbon intensity mostly used the methods of index decomposition analysis (IDA), logarithmic mean Divisia index (LMDI), Kaya, and so forth $[5,11,18,19]$. These methods are suitable to find out the driving factors of energy intensity and carbon intensity but incapable of reflecting the dynamic changes of various types of energy. The Markov model can well reflect the states of things in the development and will be conducive to studying the transition of the primary energy share. Professor Deng Julong first proposed the GM $(1,1)$ model and applied it in China's grain yield prediction $[20,21]$. The follow-up studies proved that the model has a wide range of applicability $[22,23]$. GM $(1,1)$ model is to establish the grey differential equation by using a small amount of incomplete information to describe the development law of things. Energy system has the characteristics of grey system, so it is suitable to predict the energy consumption with the grey model. This paper will mainly use the Markov model and grey model to study the change of primary energy structure and carbon emission reduction.

The rest of this paper is organized as follows. In Section 2, we present Markov chain model and GM $(1,1)$ model. In Section 3, we conduct the empirical research and analyze the obtained results. In Section 4, we provide the policy recommendations for carbon emissions reduction. And Section 5 is conclusion.

\section{Methodologies}

Markov chain prediction model, GM $(1,1)$ model, and the simple linear regression model will be used in this study. This part will focus on the modeling processes of the former two models. And the modeling process of the simple linear regression model can refer to the relevant literature [24].

\subsection{Markov Chain Model}

2.1.1. Markov Chain Prediction Principle. The Markov chain was proposed by Andre Markov in 1906 and has been widely used in natural science, engineering technology, and public 
utility [25-28]. A Markov chain can be expressed as $\mathbf{X}_{t}=$ $\mathbf{X}(t), t=0,1,2, \ldots$, and has the following property:

$$
\begin{gathered}
\mathbf{P}\{X(t+1)=j \mid X(t)=i, X(t-1)=i-1, \ldots, X(1) \\
\left.=i_{1}, X(0)=i_{0}\right\}=\mathbf{P}\{X(t+1)=j \mid X(t)=i\} .
\end{gathered}
$$

For Markov chain $\{\mathbf{X}(t), t=0,1,2, \ldots\}, P_{i j}(k)$, which is called one-step transition probability, indicates that it is in state $i$ at time $k$ and transfers to state $j$ at time $k+1$; that is,

$$
P_{i j}(k)=P\{X(k+1)=j \mid X(k)=i\} .
$$

For Markov chain $\{\mathbf{X}(t), t=0,1,2, \ldots\}, P_{i j}(k, s)$, which is called $k$-step transition probability, indicates that it is in state $i$ at time $k$ and transfers to state $j$ after $k$-step; that is,

$$
P_{i j}(k, s)=P\{X(k+s)=j \mid X(k)=i\} .
$$

The Markov chain $\{\mathbf{X}(t), t=0,1,2, \ldots\}$ is called homogeneous Markov chain, if its one-step transition probability is independent of state's time; that is,

$$
P\{X(k+1)=j \mid X(k)=i\}=P_{i j} .
$$

For homogeneous Markov chain, set its absolute probability to be $P_{j}(k), s$-step transition probability is $P_{i j}^{(s)}$, and then the following equations are established:

$$
\begin{aligned}
P_{j}(s+1) & =\sum_{i \in I} P_{i}(s) P_{i j} \\
P_{j}(s) & =\sum_{i \in I} P_{i}(0) P_{i j}^{(s)} .
\end{aligned}
$$

More generally, it is known by the Chapman-Kolmogorov equation that

$$
P_{i j}^{(m+n)}=\sum_{k \in I} P_{i k}^{(m)} P_{k j}^{(n)}
$$

These are the theoretical bases of Markov chain prediction.

2.1.2. Constructing Markov Chain Model. In this part, the Markov chain model will be constructed to predict the transition of primary energy structure. The shares of coal, oil, natural gas, and nonfossil energy (hydroelectric power, nuclear power, and wind power) in the primary energy consumption are recorded as $\mathbf{A}_{t}=\left[a_{1}(t), a_{2}(t), a_{3}(t), a_{4}(t)\right]$. Use $\mathbf{P}=\left(p_{i j}\right)_{4 \times 4}$ to indicate the transition probability from class $i$ energy to class $j$ energy one year later. $p_{i j} \geq 0$ and satisfies the following constraints:

$$
\sum_{j=1}^{4} p_{i j}=1, \quad p_{i j} \geq 0, \quad(i=1,2,3,4) .
$$

The most critical step in using Markov chain is to determine the transition probability matrix $\mathbf{P}$. This paper first established the ordinary least squares regression model of the transition probability and then estimated the transition probability according to the proportions of the four types of energies.

Assume that $\omega_{j}(k)$ represented the probability that variable $\omega$ was in state $j(j=1,2,3,4)$ at time $k$; then,

$$
\sum_{j=1}^{4} \omega_{j}(k)=1, \quad(k=0,1,2, \ldots, T) .
$$

According to the basic properties of Markov chain and $\sum_{j=1}^{4} p_{i j}=1,(i=1,2,3,4)$, the following formula was established:

$$
\omega_{j}(k)=\sum_{i=1}^{4} \omega_{i}(k-1) P_{i j}, \quad j=1,2,3,4 .
$$

Considering the error variables $u_{j}(k)$, the linear regression model of the state transition probability can be obtained:

$$
\omega_{j}(k)=\sum_{i=1}^{4} \omega_{i}(k-1) P_{i j}+u_{j}(k), \quad j=1,2,3,4 .
$$

Let

$$
\begin{aligned}
& \mathbf{W}_{j}=\left[\omega_{j}(1), \omega_{j}(2), \ldots, \omega_{j}(T)\right]^{T}, \\
& \mathbf{X}=\left[\begin{array}{cccc}
x_{1}(0) & x_{2}(0) & x_{3}(0) & x_{4}(0) \\
x_{1}(1) & x_{2}(1) & x_{3}(1) & x_{4}(1) \\
\vdots & \vdots & \vdots & \vdots \\
x_{1}(T-1) & x_{2}(T-1) & x_{3}(T-1) & x_{4}(T-1)
\end{array}\right], \\
& \mathbf{P}_{j}=\left[\begin{array}{llll}
p_{1 j} & p_{2 j} & p_{3 j} & p_{4 j}
\end{array}\right]^{T}, \\
& \mathbf{U}_{j}=\left[\begin{array}{llll}
u_{j}(1) & u_{j}(2) & u_{j}(3) & u_{j}(4)
\end{array}\right]^{T} ;
\end{aligned}
$$

then, formula (10) can be rewritten as follows:

$$
\mathbf{W}_{j}=\mathbf{X P}_{j}+\mathbf{U}_{j}, \quad j=1,2,3,4 .
$$

Two constraint conditions were added into formula (12), and the constrained ordinary least squares regression model was obtained:

$$
\begin{aligned}
\mathbf{W}_{j}= & \mathbf{X} \mathbf{P}_{j}+\mathbf{U}_{j} \\
\text { st. } & \sum_{j=1}^{4} P_{i j}=1 \\
& P_{i j} \geq 0, \quad i=1,2,3,4, \quad j=1,2,3,4 .
\end{aligned}
$$

Further, formula (13) can be transformed into the following standard quadratic programming optimization problem:

$$
\begin{array}{ll}
\min & (\mathbf{W}-\mathbf{X P})^{\prime}(\mathbf{W}-\mathbf{X P}) \\
\text { st. } & \sum_{j=1}^{4} P_{i j}=1, \quad i=1,2,3,4 \\
& P_{i j} \geq 0, \quad i=1,2,3,4, \quad j=1,2,3,4 .
\end{array}
$$


2.2. GM (1, 1) Model. GM $(1,1)$ is suitable for time series prediction with exponential trend. This method firstly establishes exponential model according to the accumulated sequence and then takes an inverse accumulated generating operation to obtain the forecasting value. This method has been widely used and has been proved feasible and effective in the energy system $[20,21,29,30]$. This paper establishes the grey forecasting model by referring to the literature [20, 21].

Set the original data as follows:

$$
x^{(0)}=\left\{x^{(0)}(1), x^{(0)}(2), \ldots, x^{(0)}(n)\right\} .
$$

Use 1-AGO algorithm to generate a first-order cumulative generation sequence:

$$
x^{(1)}=\left\{x^{(1)}(1), x^{(1)}(2), \ldots, x^{(1)}(n)\right\},
$$

where $x^{(1)}(k)=\sum_{i=1}^{k} x^{(0)}(i), k=1,2, \ldots, n$.

Build a first-order linear differential equation:

$$
\frac{d x^{(1)}}{d t}=a x^{(1)}+u
$$

By using the least square method, the parameters can be figured out:

$$
\left(\begin{array}{l}
\widehat{a} \\
\widehat{u}
\end{array}\right)=\left(\mathbf{B}^{T} \mathbf{B}\right)^{-1} \mathbf{B}^{T} \mathbf{Y}_{n},
$$

where

$$
\begin{aligned}
& \mathbf{Y}_{n}=\left(\begin{array}{c}
x^{(0)}(2) \\
x^{(0)}(3) \\
\vdots \\
x^{(0)}(n)
\end{array}\right), \\
& \mathbf{B}=\left(\begin{array}{cc}
-\frac{1}{2}\left[x^{(1)}(1)+x^{(1)}(2)\right] & 1 \\
-\frac{1}{2}\left[x^{(1)}(2)+x^{(1)}(3)\right] & 1 \\
\vdots & \vdots \\
-\frac{1}{2}\left[x^{(1)}(n-1)+x^{(1)}(n)\right] & 1
\end{array}\right) .
\end{aligned}
$$

Let the parameters of $\widehat{a}$ and $\widehat{u}$ return back to the differential equation, and then get the following equation:

$$
\begin{aligned}
x^{(1)}(k+1)=\left[x^{(1)}(1)-\frac{\widehat{u}}{\widehat{a}}\right] e^{-\widehat{a} k}+\frac{\widehat{u}}{\widehat{a}} & \\
& (k=0,1,2, \ldots) .
\end{aligned}
$$

Through inverse accumulated generating operation, the following grey prediction model can be got:

$$
\begin{array}{r}
\hat{x}^{(0)}(k)=\left(1-e^{\widehat{a}}\right)\left[x^{(0)}(1)-\frac{\widehat{u}}{\hat{a}}\right] e^{-\widehat{a}(k-1)} \\
\quad(k=1,2,3, \ldots),
\end{array}
$$

where $\hat{x}^{(0)}(k)$ is the estimated value of $x^{(0)}(k)$.
TABLE 1: Chinese primary energy structure (unit: \%, Mtoe).

\begin{tabular}{cccccc}
\hline Time & Crude oil & Natural gas & Coal & Nonfossil fuels & Total \\
\hline 2003 & 22.1 & 2.4 & 69.3 & 6.1 & 1204.2 \\
2004 & 22.4 & 2.5 & 68.7 & 6.4 & 1423.5 \\
2005 & 20.9 & 2.6 & 69.9 & 6.5 & 1566.7 \\
2006 & 20.4 & 2.9 & 70.2 & 6.4 & 1729.8 \\
2007 & 19.5 & 3.4 & 70.5 & 6.7 & 1862.8 \\
2008 & 18.8 & 3.6 & 70.2 & 7.4 & 2002.5 \\
2009 & 17.7 & 3.7 & 71.2 & 7.4 & 2187.7 \\
2010 & 17.6 & 4.0 & 70.5 & 7.9 & 2432.2 \\
2011 & 17.7 & 4.5 & 70.4 & 7.4 & 2613.2 \\
2012 & 17.7 & 4.7 & 68.5 & 9.1 & 2735.2 \\
2013 & 17.8 & 5.1 & 67.5 & 9.6 & 2852.4 \\
2014 & 17.5 & 5.6 & 66.0 & 10.9 & 2972.1 \\
\hline
\end{tabular}

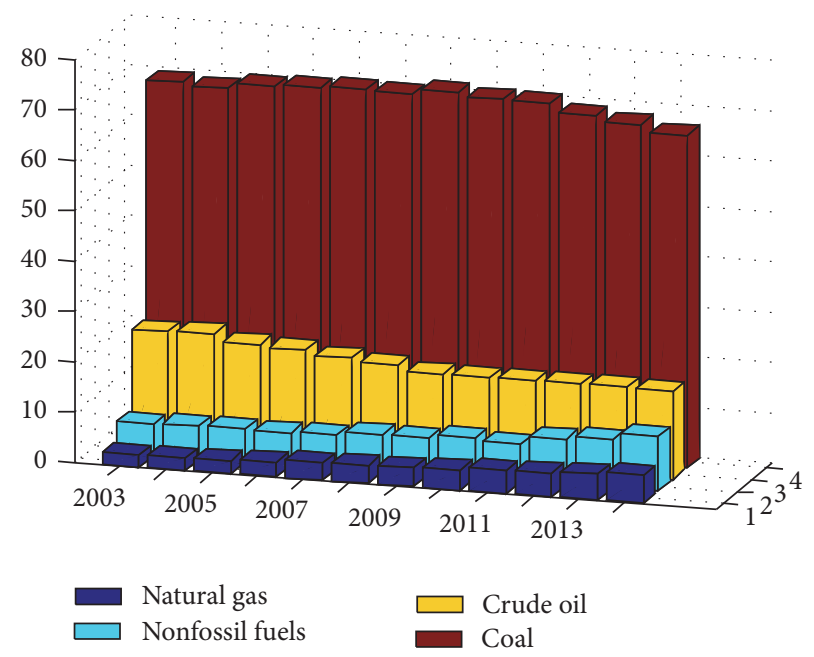

FIGURE 1: Energy consumption shares of the primary energy in China.

\section{Results and Discussions}

3.1. Data Selections and Preliminary Analysis. We chose data from the "China Statistical Yearbook," which were released by China's National Bureau of Statistics [31]. Data (from 2003 to 2014) shown in Table 1 include the shares of crude oil, natural gas, coal, and nonfossil energy, as well as the total consumption of each type of energy.

The average shares of crude oil, natural gas, coal, and nonfossil fuels from 2003 to 2014 were $19.2 \%, 3.8 \%, 69.4 \%$, and $7.7 \%$, respectively.

Figure 1 reflects the gradual increase in the shares of natural gas and nonfossil fuels and gradual decrease in the share of crude oil from 2003 to 2014. Share of coal consumption reaches its peak in 2009 and then gradually reduces. From Figure 1, we can see directly that the consumption of coal is in the dominant position of the primary energy mix in China. 


\subsection{Results and Analysis}

(a) Analysis of Target 1. By using ordinary least squares method and formula (14), the transition probabilities between the four kinds of primary energies were calculated as follows:

$$
\mathbf{P}=\left[\begin{array}{cccc}
0.7973 & 0 & 0.2027 & 0 \\
0 & 0.5244 & 0 & 0.4756 \\
0.0501 & 0 & 0.9395 & 0.0104 \\
0 & 0.2715 & 0 & 0.7285
\end{array}\right]
$$

After being determined, the state transition matrix $\mathbf{P}$ can be used to predict the structures of the primary energy in 2020 and 2030. On the basis of 2014, let $\mathbf{A}_{2014}$ denote the vector of the primary energy structure in 2014; then we can get the following formula:

$$
\mathbf{A}_{t}=\mathbf{A}_{2014} \times \mathbf{P}^{t-2014} \text {. }
$$

Inputting the values of $\mathbf{A}_{2014}=[17.5,5.6,66.0,10.9]$ and $\mathbf{P}$ into formula (23), we can obtain the prediction results: $\mathbf{A}_{2020}=[16.35,7.14,63.11,13.40]$ and $\mathbf{A}_{2030}=[14.99,9.47$, $58.14,17.40]$.

The Chinese government has formulated the nonfossil energy development plan: by 2020 and 2030, the proportions of nonfossil energy will account for $15 \%$ and $20 \%$ in primary energies consumption, respectively. The forecast results indicate that the proportion of nonfossil energy will reach $13.4 \%$ by 2020 and $17.4 \%$ by 2030 . That is to say, target 1 cannot be achieved, if the current energy consumption pattern is not changed.

(b) Analysis of Target 2. Using GM $(1,1)$ model and simple linear regression method (SLRM), the energy consumptions in 2020 and 2030 were predicted.

For the GM $(1,1)$ model, we used the data from 2010 to 2014 to estimate the parameters and obtained the prediction equation as follows:

$$
\widehat{x}^{(0)}(k)=2507.02 \times e^{0.04(k-1)} \quad(k=1,2,3, \ldots),
$$

where $\widehat{\alpha}=-0.04, \widehat{\mu}=2457.04$. The average relative error of GM $(1,1)$ model is $0.11 \%$.

For the simple linear regression method (SLRM), we used the data from 2003 to 2014 to estimate the parameters and obtained the prediction equation as follows:

$$
y=-326510+163.63 x, \quad x=2003,2004, \ldots,
$$

where $y$ represents the primary energy consumption and $x$ indicates the year to forecast $a=-326510, b=163.63, R^{2}=$ $0.9953, P=5.75 \times 10^{-13}<0.05$.

These two models were used to fit the historical data, and the results were shown in Figure 2.

From Figure 2, we can see that the historical data are fitted with a very high accuracy, as confirmed by the correctness and availability of the two models. The prediction results

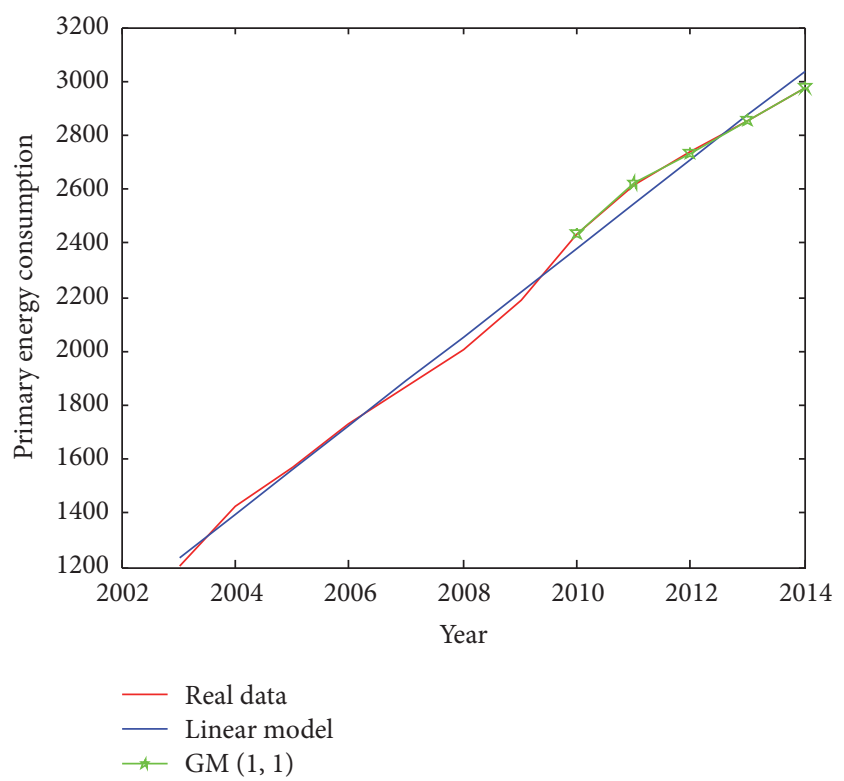

Figure 2: The models' fitting results.

of the energy consumption in 2020 and 2030 by these two models were shown in Table 2.

The average of the predicted values was treated as an acceptable estimate value. So, considering the shares of the four primary energies in 2020 and 2030, the energy consumptions can be worked out. According to the IPCC recommended crude oil emission coefficient $\left(3.096 \mathrm{kgCO}_{2} / \mathrm{kg}\right)$, the $\mathrm{CO}_{2}$ emissions can be calculated in 2020 and 2030, which are 12162.2 Mt and 17865.8 Mt, respectively.

The Chinese authorities' commitment to carbon emissions reduction was to achieve the peak of 12 billion tons of carbon emissions by 2030. However, the calculation results show that if the current development trend does not change, then China's carbon emissions will outnumber 12 billion tons before 2020 and will continue to increase. Clearly, China cannot achieve the promised targets.

(c) Analysis of Target 3. According to the data released by China's National Bureau of Statistics, China's energy consumption per 10 thousand yuan is 1.276 tons of standard coal in 2005 [31]. According to IPCC's proposed carbon emissions coefficient $\left(2.46 \mathrm{kgCO}_{2} / \mathrm{kg}\right.$ standard coal), we can calculate that the coefficient of carbon emissions intensity in 2005 is 3.14. In addition, China's GDP in 2015 is 67.6708 trillion yuans.

\section{Parameters Description}

$\alpha_{t}$ : carbon emissions intensity in year $t$

$c_{t}$ : carbon dioxide emissions in year $t$

$\operatorname{gdp}_{t}$ : gross domestic product in year $t$

$r$ : average annual growth rate of GDP

$\lambda$ : percentage of carbon emissions reduction 
TABLE 2: Primary energy consumption forecast results (unit: Mtoe).

\begin{tabular}{lccccccc}
\hline Time & GM $(1,1)$ & SLRM & Average & Crude oil & Natural gas & Coal & Nonfossil energy \\
\hline 2020 & 3843.2 & 4013.5 & 3928.35 & 642.3 & 280.5 & 2479.2 & 526.4 \\
2030 & 5891.4 & 5649.8 & 5770.6 & 865.0 & 546.5 & 3355.0 & 1004.0 \\
\hline
\end{tabular}

According to the concepts of carbon intensity and carbon emissions reduction, the following formula can be got:

$$
\begin{aligned}
& \lambda=\frac{\alpha_{t}-c_{t} / \mathrm{gdp}_{t}}{\alpha_{t}} \\
& \lambda=\frac{\alpha_{2005}-c_{2020} / \operatorname{gdp}_{2015}(1+r)^{5}}{\alpha_{2005}} \\
& r=\sqrt[5]{\frac{c_{2020}}{\alpha_{2005}(1-\lambda) \operatorname{gdp}_{2015}}}-1 .
\end{aligned}
$$

As known, $\alpha_{2005}=3.14$, let $r=0$, and then $\lambda=0.428$. This means that, with the current carbon emissions trend, even if the average annual growth rate of GDP from 2015 to 2020 is equal to zero, carbon emissions intensity in 2020 can still be reduced by $42.8 \%$ compared with the 2005 level. Even to achieve the upper limit of $45 \%$, the average annual growth rate of GDP from 2015 to 2020 is only about $0.8 \%$. Similarly, let $\lambda=65 \%$; then $r=6.02 \%$. This shows that if the target of reducing carbon emissions by $65 \%$ is achieved, the average annual growth rate of GDP from 2015 to 2030 must reach $6.02 \%$.

Through the above analysis, we can know that the target to reduce carbon emissions by $40-45 \%$ in 2020 compared with the 2005 level can be easily reached, almost without any effort. Related studies have also drawn similar conclusions $[4,17]$. However, the target of $65 \%$ carbon emissions reduction in 2030 compared with the 2005 level requires a comprehensive plan to achieve. In summary, target 3 has partial constraint.

\section{Policy Recommendations}

Some new ideas, which guide China's development, were put forward in the 13th Five-Year Plan, such as promoting the energy revolution, changing the patterns of energy production and utilization, optimizing the energy supply structure, improving energy efficiency, and constructing clean and lowcarbon energy system [32]. "Energy Development Strategic Action Plan (2014-2020)," issued by China's State Council in 2014, required to speed up the construction of clean, efficient, safe, and sustainable energy system [33]. Moreover, "Paris Agreement" officially entered into force in November 4, 2016, and the Chinese government's emission reduction responsibility would be subject to legal constraints. In view of the high carbon energy structure, China's State Council has announced that the national carbon emissions trading market will be started in 2017, in order to control the total amount of coal consumption.

Meanwhile, from the above analysis, we can know that the significant characteristics of China's energy structure are as follows. (1) Coal has a dominant position. Coal consumption accounts for about $60 \%$ of the total energy consumption, and $\mathrm{CO}_{2}$ emissions related to coal combustion account for the vast majority in the total emissions. (2) The proportion of nonfossil energy is low. The conclusion can be drawn from the calculation results that the preset targets of $15 \%$ and $20 \%$ cannot be achieved by 2020 and 2030 without human intervention. (3) The energy efficiency is relatively low. Energy efficiencies in many sectors are generally lower and energy intensities are generally higher than that of developed countries. (4) Science and technology innovation in carbon emission reduction should be strengthened. Although China has made considerable progress in science and technology, but, in terms of carbon emission reduction science and technology, there is still a great room to improve.

In order to achieve the emission reduction targets, China must improve the primary energy structure and must take the road of low-carbon energy. Therefore, combined with China's national conditions, this paper puts forward the following energy policy recommendations.

4.1. Controlling Coal Demands Moderately. The characteristics of energy resources in China determine the long-term energy production and consumption based on coal. In the thermal power industry, the carbon emissions from coal combustion are more $23-31 \%$ and $70-80 \%$ than that of the combustion of the same calorific value of oil and natural gas. The higher the proportion of coal in primary energy structure, the greater the amount of $\mathrm{CO}_{2}$ emissions in the energy activities. Therefore, controlling coal demand and reducing the proportion of coal are of special significance for China to improve the primary energy structure.

4.2. Developing Nonfossil Energies. The low-carbon energies need to strive to develop in China, including natural gas, hydropower, nuclear power, and nonhydropower renewable energies.

(a) Natural Gas. Natural gas is one of fossil fuels, with less pollutant emissions compared with coal. In the medium-long term, natural gas will be the fastest growing energy in China's energy structure and it becomes an important force in the development of China's low-carbon energies. Unfortunately, China lacks natural gas. Therefore, China should strengthen international cooperation in the energy field and look to Africa, Russia, Central Asia, and even Canada as possible alternative sources of its gas imports.

(b) Hydropower. Hydropower belongs to nonfossil energy, and it is technically matured and suitable for large-scale development in China. In order to achieve the share of $15 \%$ for nonfossil energy in primary energy, hydropower installed capacity should reach 340 million kW by 2020 . 
(c) Nuclear Power. Compared with traditional fossil fuels, nuclear power is a kind of clean energy, without greenhouse gas emissions in its power generation process. Affected by the Fukushima nuclear accident in Japan, China once slowed down the development of nuclear power. In fact, nuclear power in China should be developed vigorously and constitute a "troika" with natural gas and hydropower together to promote the development of China's low-carbon energy.

(d) Nonhydropower Renewable Energies. In the medium-long term, renewable energies such as wind energy, solar energy, and biomass energy are important measures to improve the energy structure and reduce the environmental pollution. China should gradually improve the energy law and policies system and create a favorable environment for the development of low-carbon energies.

4.3. Improving Energy Efficiency. Energy efficiency improvement is still an important measure for carbon reduction in China. China should establish the long-term mechanism of energy-saving and further eliminate outdated production capacity in high energy consuming industries; at the same time, China should optimize the industrial structure and improve the technology to promote energy efficiency, especially in the transportation, construction industry, power industry, and so on.

4.4. Enhancing the Ability of Innovation. Currently, the world's leading energy research institutions, including the IEA, have agreed to carbon capture and storage (CCS) technology as the main technical means of future carbon emissions reduction. At present, reasons that China's CCS technology is difficult to apply are the high energy consumption and high cost. China should attach great importance to the development of CCS technology, strengthen international cooperation, continuously track the latest progress of CCS technology, and let CCS be more widely used as early as possible.

\section{Conclusions}

Today, carbon emission reduction has become a realistic problem that must be faced seriously for China. It has become an urgent task for the Chinese government to improve primary energy structure and develop low-carbon energy. This paper constructed the Markov chain model and used it to predict the primary energy shares in 2020 and 2030 and accordingly determined that the nonfossil energy development target which Chinese authorities proposed cannot be achieved without extra effort. This paper built the GM (1, 1) model and simple linear regression model, used them to predict energy consumption in 2020 and 2030, calculated the amount of energy carbon emissions in China, and determined that the carbon peak objective which Chinese authorities set cannot be achieved naturally. This paper estimated the carbon emission intensities of China in 2020 and 2030 and drew the conclusion that the carbon emission intensity target proposed by the Chinese government had only partial constraint. Considering China's current national conditions, this paper put forward that the key measures to achieve China's carbon emissions reduction were to control coal demand, develop low-carbon energy, improve energy efficiency, and enhance the ability of innovation in carbon emission reduction field.

\section{Competing Interests}

The authors declare that there are no competing interests regarding the publication of this paper.

\section{Acknowledgments}

This work was supported by the Fundamental Research Funds for the Central Universities (2015MS150) and the National Natural Science Foundation of China (Grant no. 71373067).

\section{References}

[1] IEA (International Energy Agency), $\mathrm{CO}_{2}$ Emissions from Fuel Combustion: Highlight, OECD/IEA, Paris, France, 2011.

[2] UNFCCC (United Nations Framework Convention on Climate Change), Text of the Kyoto Protocol, http://unfccc.int/ key_documents/kyoto_protocol/items/6445.php.

[3] The Climate Group, "China to cut emissions intensity by 60 65\% from 2005 level by 2030: INDC," http://www.theclimategroup.org/what-we-do/news-and-blogs/china-to-cut-emissionsby-60-65-from-2005-level-indc/.

[4] B. W. Yi, J. H. Xu, and Y. Fan, "Determining factors and diverse scenarios of $\mathrm{CO}_{2}$ emissions intensity reduction to achieve the $40-45 \%$ target by 2020 in China-a historical and prospective analysis for the period 2005-2020," Journal of Cleaner Production, vol. 122, pp. 87-101, 2016.

[5] B. W. Ang and B. Su, "Carbon emission intensity in electricity production: a global analysis," Energy Policy, vol. 94, pp. 56-63, 2016.

[6] X. L. Wang and B. Q. Lin, "How to reduce $\mathrm{CO}_{2}$ emissions in China's iron and steel industry," Renewable and Sustainable Energy Reviews, vol. 57, pp. 1496-1505, 2016.

[7] K. Li and B. Lin, "China's strategy for carbon intensity mitigation pledge for 2020: evidence from a threshold cointegration model combined with Monte-Carlo simulation methods," Journal of Cleaner Production, vol. 118, pp. 37-47, 2016.

[8] X. Yuan, L. Li, H. Gou, and T. Dong, "Energy and environmental impact of battery electric vehicle range in China," Applied Energy, vol. 157, pp. 75-84, 2015.

[9] H.-Q. Li, L.-M. Wang, L. Shen, and F.-N. Chen, "Study of the potential of low carbon energy development and its contribution to realize the reduction target of carbon intensity in China," Energy Policy, vol. 41, pp. 393-401, 2012.

[10] J. Jewell, A. Cherp, and K. Riahi, "Energy security under decarbonization scenarios: an assessment framework and evaluation under different technology and policy choices," Energy Policy, vol. 65, pp. 743-760, 2014.

[11] J. C. Steckel, M. Jakob, R. Marschinski, and G. Luderer, "From carbonization to decarbonization? Past trends and future scenarios for China's $\mathrm{CO}_{2}$ emissions," Energy Policy, vol. 39, no. 6, pp. 3443-3455, 2011. 
[12] J.-L. Jiao, Y.-Y. Qi, Q. Cao, L.-C. Liu, and Q.-M. Liang, “China's targets for reducing the intensity of $\mathrm{CO}_{2}$ emissions by 2020," Energy Strategy Reviews, vol. 2, no. 2, pp. 176-181, 2013.

[13] Y. Y. Lu, A. Stegman, and Y. Y. Cai, "Emissions intensity targeting: from China's 12th Five Year Plan to its Copenhagen commitment," Energy Policy, vol. 61, pp. 1164-1177, 2013.

[14] R. Wang, W. Liu, L. Xiao, J. Liu, and W. Kao, "Path towards achieving of China's 2020 carbon emission reduction targeta discussion of low-carbon energy policies at province level," Energy Policy, vol. 39, no. 5, pp. 2740-2747, 2011.

[15] D. I. Stern and F. Jotzo, "How ambitious are China and India's emissions intensity targets?" Energy Policy, vol. 38, no. 11, pp. 6776-6783, 2010.

[16] H. C. Dai, T. Masui, Y. Matsuoka, and S. Fujimori, "Assessment of China's climate commitment and non-fossil energy plan towards 2020 using hybrid AIM/CGE model," Energy Policy, vol. 39, no. 5, pp. 2875-2887, 2011.

[17] J. M. Cansino, R. Román, and J. M. Rueda-Cantuche, "Will China comply with its 2020 carbon intensity commitment?" Environmental Science \& Policy, vol. 47, pp. 108-117, 2015.

[18] J. Jiang, "China’s urban residential carbon emission and energy efficiency policy," Energy, vol. 109, pp. 866-875, 2016.

[19] B. W. Ang and G. Pandiyan, "Decomposition of energy-induced $\mathrm{CO}_{2}$ emissions in manufacturing," Energy Economics, vol. 19, no. 3, pp. 363-374, 1997.

[20] J. L. Deng, “Grey control system," Journal of Huazhong University of Science and Technology, no. 3, pp. 9-18, 1982.

[21] J. L. Deng, "Grey fuzzy forecast and control for grain," Journal of Huazhong University of Science and Technology, no. 2, pp. 1-8, 1983.

[22] J. Wang, H. Jiang, Q. Zhou, J. Wu, and S. Qin, "China's natural gas production and consumption analysis based on the multicycle Hubbert model and rolling Grey model," Renewable and Sustainable Energy Reviews, vol. 53, pp. 1149-1167, 2016.

[23] L. Wu, S. Liu, D. Liu, Z. Fang, and H. Xu, "Modelling and forecasting $\mathrm{CO}_{2}$ emissions in the BRICS (Brazil, Russia, India, China, and South Africa) countries using a novel multi-variable grey model," Energy, vol. 79, pp. 489-495, 2015.

[24] D. C. Montgomerie, Introduction to Linear Regression Analysis, Machinery Industry Press, Beijing, China, 5th edition, 2016.

[25] J. Liisberg, J. K. Møller, H. Bloem, J. Cipriano, G. Mor, and H. Madsen, "Hidden Markov Models for indirect classification of occupant behaviour," Sustainable Cities and Society, vol. 27, pp. 83-98, 2016.

[26] N.-B. Zhao, J.-L. Yang, S.-Y. Li, and Y.-W. Sun, "A GM $(1,1)$ Markov chain-based aeroengine performance degradation forecast approach using exhaust gas temperature," Mathematical Problems in Engineering, vol. 2014, Article ID 832851, 11 pages, 2014.

[27] Z.-L. Shen, T.-Z. Huang, B. Carpentieri, and C. Wen, "A costeffective smoothed multigrid with modified neighborhoodbased aggregation for Markov Chains," Mathematical Problems in Engineering, vol. 2015, Article ID 816935, 15 pages, 2015.

[28] G. Casale, A. Sansottera, and P. Cremonesi, "Compact Markovmodulated models for multiclass trace fitting," European Journal of Operational Research, vol. 255, no. 3, pp. 822-833, 2016.

[29] P.-P. Xiong, Y.-G. Dang, T.-X. Yao, and Z.-X. Wang, "Optimal modeling and forecasting of the energy consumption and production in China," Energy, vol. 77, pp. 623-634, 2014.
[30] C. Yuan, S. Liu, and Z. Fang, "Comparison of China's primary energy consumption forecasting by using ARIMA (the autoregressive integrated moving average) model and GM(1,1) model," Energy, vol. 100, pp. 384-390, 2016.

[31] NBS (National Bureau of Statistics of the People's Republic of China), Chinastatistics Yearbook (2004-2015), China Statistics Press, Beijing, China, 2015.

[32] State Council of the PRC, "The 13th Five-Year Plan," http:// www.sh.xinhuanet.com/2016-03/18/c_135200400_2.htm.

[33] State Council of the PRC, "Energy development strategic action plan (2014-2020)," http://www.chinanews.com/ny/2014/11-20/ 6795577.shtml. 


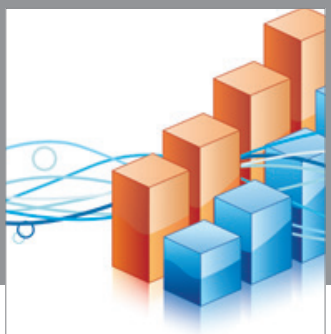

Advances in

Operations Research

vatem alat4

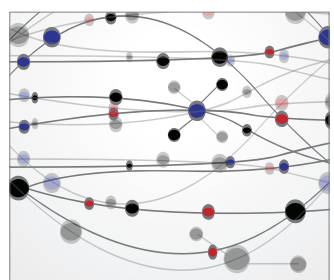

\section{The Scientific} World Journal
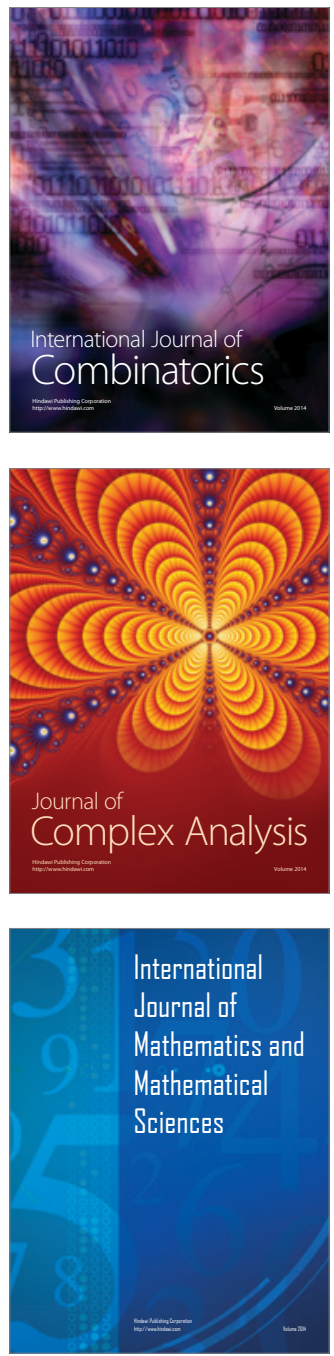
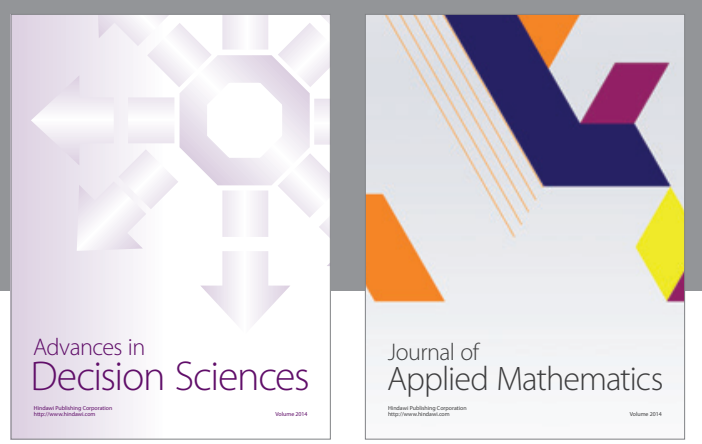

Algebra

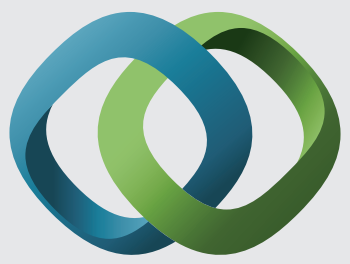

\section{Hindawi}

Submit your manuscripts at

http://www.hindawi.com
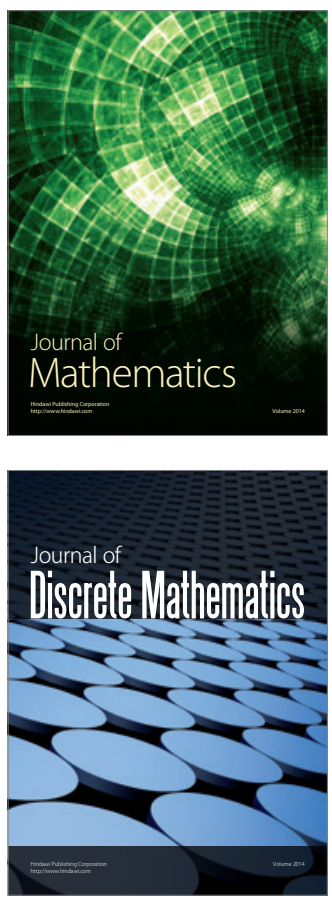

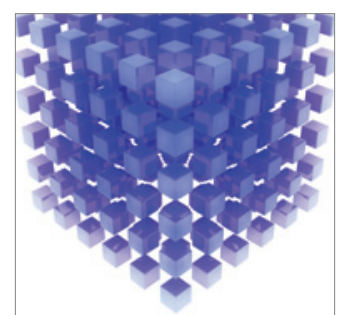

Mathematical Problems in Engineering
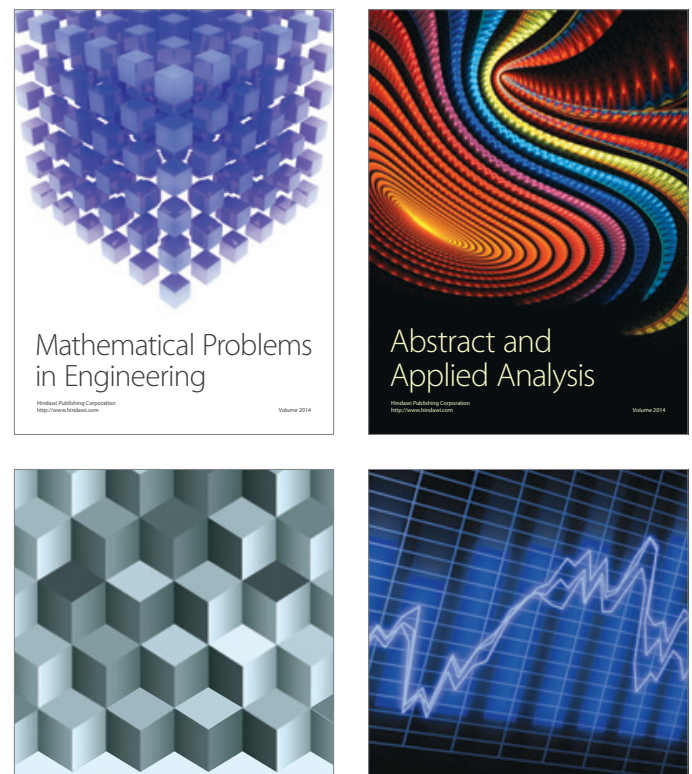

Journal of

Function Spaces

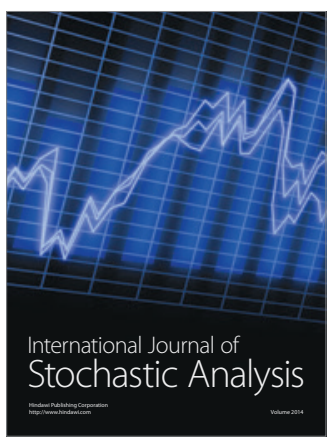

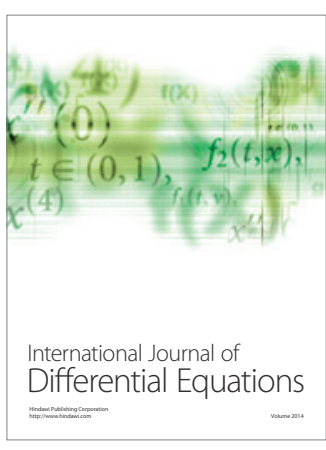
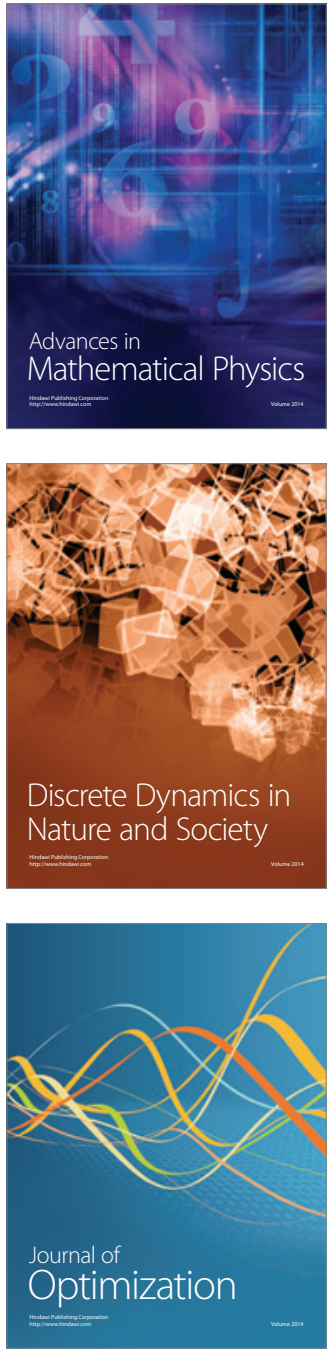